\title{
Nationale Verpflichtungen auf Grundlage des Pariser Klimaabkommens
}

\author{
Coordinating Lead Authors (CLAs) \\ Franz Prettenthaler \\ Lead Authors (LAs) \\ Franz Prettenthaler, Andrea Damm, Stefan Gössling, Christoph Neger \\ Contributing Authors (CAs) \\ Stephan Schwarzinger, Willi Haas
}

Kap. 11 hat gezeigt, dass es derzeit noch große Unsicherheiten darüber gibt, wie hoch tatsächlich der Beitrag des Tourismus zu den nationalen und internationalen Treibhausgasemissionen ist. Es ist jedoch gewiss, dass der Anteil bedeutend ist und, in Zusammenhang mit dem prognostizierten Wachstum des weltweiten Tourismus, wahrscheinlich weiter steigen wird. Daher stellt sich die Frage, wie die Emissionen der touristischen Aktivitäten gesenkt werden könnten. Im vorliegenden Kapitel wird berichtet, welche Verpflichtungen und Strategien es dazu aktuell auf politischer Ebene gibt, beginnend mit internationalen Verträgen, insbesondere dem Pariser Klimaschutzabkommen, und Vorgaben der Europäischen Union, bei denen Österreich sich verpflichtet hat, teilzunehmen. In weiterer Folge richtet sich das Augenmerk auf eine detaillierte Betrachtung und Bewertung der Klimaschutz-, Klimawandelanpassungs- und Tourismusstrategien auf Bundesebene und in den einzelnen Bundesländern. Im Anschluss daran werden allgemein mögliche Maßnahmen und Potenziale besprochen. Schließlich wird unter dem Schlagwort „Paris Lifestyle“ diskutiert, welche Maßnahmen notwendig sind, um auf individueller Ebene zum Ziel eines $\mathrm{CO}_{2}$-armen bzw. -neutralen Tourismus beitragen zu können. Dazu werden auch Beispiele innovativer, emissionsarmer Angebote von Tourismusbetrieben und Destinationen vorgestellt.

\subsection{Derzeitige nationale Ambitionen und international gemachte Zusagen}

Seit Anfang der 1990er-Jahre wird global über eine gemeinsam abgestimmte Klimapolitik diskutiert. Im Jahr 1994 trat das Rahmenübereinkommen der Vereinten Nationen in Kraft, dessen Mitgliedsstaaten sich fortan an einer jährlich veranstalteten Konferenz beteiligten. In dem 1997 erarbeiteten Kyotoprotokoll verpflichteten sich die beteiligten Länder erstmals auf internationaler Ebene vertraglich zu Reduktionszielen für
Treibhausgasemissionen. Am 12.12.2015 wurde dann schließlich auf der Weltklimakonferenz in Paris das Pariser Klimaabkommen als jüngster Meilenstein in der internationalen Klimapolitik beschlossen, welches sowohl aufgrund der breiten Beteiligung als auch wegen seiner ambitionierten Zielsetzungen als von historischer Bedeutung gilt (UNFCCC o.J.).

Wichtigstes Ziel des Abkommens ist, dass der globale Temperaturanstieg unter $2^{\circ} \mathrm{C}$ über dem vorindustriellen Niveau bleibt, nach Möglichkeit sogar nur auf $1,5^{\circ} \mathrm{C}$ begrenzt wird. Dadurch sollen, gemäß den Berechnungen des IPCC, stärkere negative Auswirkungen des Klimawandels verhindert werden. Neben diesem prioritären Ziel sind im Abkommen auch Maßnahmen zur Anpassung an den Klimawandel enthalten sowie zur Unterstützung von Entwicklungsländern beim Erreichen ihrer Mitigations- und Anpassungsziele. Die Vertragsstaaten sind dazu aufgerufen, regelmäßig ihre nationalen Klimaschutzziele bekanntzugeben (Nationally Determined Contributions - NDCs), mit einer jedes Mal stärkeren Reduktion der Treibhausgasemissionen (Schleussner et al. 2016; UNFCCC o.J.). Die Europäische Union ist an dem Abkommen, welches im November 2016 in Kraft trat, geschlossen beteiligt (Europäische Kommission 2016a). Die österreichische Klimapolitik muss sich daher an den gemeinsamen Vorgaben auf europäischer Ebene ausrichten.

\subsubsection{Klimapolitik der Europäischen Union}

Die EU gab bereits vor den Verhandlungen in Paris bekannt, dass die Mitgliedsstaaten sich bis $2030 \mathrm{zu}$ einer Reduktion der Treibhausgasemissionen um 40 \% im Vergleich zu 1990 verpflichten werden, zudem sollte die Energieeffizienz im selben Zeitraum um $27 \%$ verbessert werden und der Anteil erneuerbarer Energie auf $27 \%$ angehoben werden (Lettische EU-Ratspräsidentschaft und Europäische Kommission 2015). Die beiden letzteren Zielvorgaben wurden inzwischen auf 
$32,5 \%$ bzw. $32 \%$ angehoben (Europäische Kommission 2018). In dem 2015 verfassten Positionspapier werden auch die Sektoren erwähnt, welche schwerpunktmäßig zu dieser Reduktion beitragen sollen. Es sind dies Energie, Industrie, Landwirtschaft, Abfall sowie Landnutzung und Forstwirtschaft. Trotz der Bedeutung der Tourismuswirtschaft für die Treibhausgasemissionen auf internationaler Ebene (siehe Kap. 11) wird diese nicht explizit als Themenschwerpunkt behandelt. Der Tourismus ist aber indirekt sehr wohl betroffen, da Touristen und touristische Betriebe Produkte aus industrieller und landwirtschaftlicher Produktion nutzen, Abfall produzieren und Landflächen in Anspruch nehmen. Am stärksten ist der Bezug zum Tourismus jedoch eindeutig im Bereich Energie, sowohl was touristische Mobilität als auch den Energieverbrauch touristischer Betriebe angeht.

In diesem Zusammenhang hat die Europäische Kommission 2016 ein Maßnahmenpaket unter dem Titel „Saubere Energie für alle Europäer" gestartet (Europäische Kommission 2018). Darin enthalten sind Initiativen zu unterschiedlichen Themenbereichen, welche teilweise noch verhandelt werden und teilweise bereits vom Europäischen Parlament und vom EU-Rat verabschiedet wurden und nun rechtsgültig sind (Stand September 2018). Neben den Bereichen erneuerbare Energien, Energieeffizienz allgemein, Governance der Energieunion und Gestaltung des Elektrizitätsmarkts ist dabei auch die Energieeffizienz von Gebäuden ein wichtiger Punkt. Bereits 2010 war vereinbart worden, dass alle Neubauten ab 2020 Niedrigstenergiegebäude sein müssen (Europäisches Parlament und Rat der Europäischen Union 2010). Nun sollen die Mitgliedsstaaten vor allem ihre Anstrengungen verstärken, um bis 2050 einen weitgehend emissionsfreien Gebäudebestand zu erreichen, u. a. durch eine langfristige, mit ausreichenden Mitteln ausgestattete Strategie zur Sanierung älterer Gebäude. Des Weiteren sollen Gebäude über Infrastruktur zur Unterstützung von E-Mobilität verfügen und intelligente Technologien sollen verstärkt für einen effizienten Gebäudebetrieb sorgen. Spezifische Maßnahmen für den Tourismus sind im Paket „Saubere Energie für alle Europäer“ nicht enthalten (Europäische Kommission 2018).

Daneben ist auch die europäische Strategie für emissionsfreie Mobilität erwähnenswert (Europäische Kommission 2016b), welche einige aus touristischer Sicht interessante Punkte enthält. So sollen Maßnahmen gesetzt werden, um die Emissionen im Luftverkehr zu reduzieren (Ziel ist ein emissionsfreies Wachstum des Luftfahrtsektors ab 2020), der Schienenverkehr soll durch einen geänderten Rechtsrahmen für den Eisenbahnsektor wettbewerbsfähiger und für Fahrgäste attraktiver werden und im Bereich E-Mobilität soll eine europaweit verfügbare Lade-, Betankungs- und Wartungsinfrastruktur aufgebaut werden, mit dem Ziel „... eine Autoreise quer durch Europa unternehmen zu können, bei der das Aufladen des Fahrzeugs ebenso problemlos ist wie ein konventioneller Tankvorgang“ (Europäische Kommission 2016b, S. 6).
Schließlich gibt es auf EU-Ebene auch eine Klimawandelanpassungsstrategie, welche bereits 2013 von der Kommission veröffentlicht und inzwischen noch nicht erneuert wurde (Stand September 2018). Auch hierbei ist der Tourismus kein Schwerpunktthema. Das Strategiepapier enthält jedoch Guidelines für die Ausarbeitung von Anpassungsstrategien auf Ebene der Mitgliedsstaaten, bei denen der Tourismus als einer der vom Klimawandel betroffenen Sektoren erwähnt wird (Europäische Kommission 2013).

\subsubsection{Klimarelevante Aspekte der europäischen Tourismuspolitik}

Die Europäische Kommission startete 2010 eine neue Strategie zur Entwicklung des Tourismus in der EU, wobei in zwei von vier genannten Schwerpunkten der Strategie neben Qualitätsoptimierung der Aspekt der Nachhaltigkeit genannt wird, sowohl für den europäischen Tourismus generell als auch speziell für einzelne Destinationen. Konkret gibt es drei Aktionsbereiche für nachhaltigen Tourismus, die auch klimaschutzrelevante Maßnahmen beinhalten. Die Initiative Sustainable Transnational Tourism Products förderte von 2011 bis 2015 länderübergreifende Projekte zur Entwicklung nachhaltiger Tourismusangebote, wie zum Beispiel Fahrradrouten oder Naturtourismusprodukte (Europäische Kommission 2019a).

Der zweite Aktionsbereich betrifft die Entwicklung des European Tourism Indicators System (ETIS), welches den Entscheidungsträgern in europäischen Tourismusdestinationen dabei helfen soll, ihre Nachhaltigkeitsperformance zu messen und dementsprechende Maßnahmen zu ergreifen. Dazu wurden 41 Indikatoren definiert, wobei zwei speziell auf die Thematik Klimawandel abzielen („Prozentsatz der Tourismusbetriebe, die in Programme zum Klimaschutz und in Anpassungsaktionen involviert sind“, sowie „Prozentsatz von touristischer Infrastruktur in gefährdeten Zonen“), dazu gibt es vier Indikatoren betreffend die Reduktion von Transportemissionen und drei Indikatoren zum Energieverbrauch (Energieeffizienz und Nutzung erneuerbarer Energieträger; Europäische Kommission 2016c).

Als dritter Aktionsbereich können EMAS (EU EcoManagement and Audit Scheme), ein Programm mit dem Unternehmen dabei unterstützt werden, ihre Umweltbilanz zu verbessern, sowie das Zertifizierungsprogramm EU Ecolabel für umweltfreundliche Unternehmen genannt werden (Europäische Kommission 2019a). Letzteres beinhaltet als spezielle Produktkategorie die touristischen Beherbergungsbetriebe, wobei unter anderem die Bereiche energieeffiziente Heizung, Kühlung und Beleuchtung, der Bezug elektrischer Energie aus erneuerbaren Quellen und die Bewerbung einer umweltfreundlichen Anreise bewertet werden (Europäische Kommission 2019b). 


\subsection{3 Österreichische Klimapolitik}

Infolge der Ratifizierung des Pariser Klimaabkommens und im Einklang mit der europäischen Klimapolitik stellte die österreichische Bundesregierung im Juni 2018 die finale Version einer österreichischen Klima- und Energiestrategie vor (BMNT und BMVIT 2018). Die darin enthaltenen Vorgaben sind hauptsächlich auf das Jahr 2030 und teilweise auch auf 2050 ausgerichtet, weshalb die Strategie den Titel \#mission2030 trägt. Die übergeordneten Ziele der Strategie sind unter den Schlagwörtern ökologische Nachhaltigkeit, Versorgungssicherheit und Wettbewerbsfähigkeit/Leistbarkeit zusammengefasst.

Im Bereich der ökologischen Nachhaltigkeit sollen die Treibhausgasemissionen bis 2030 um $36 \%$ gegenüber 2005 gesenkt werden. Für Wirtschaftssektoren, welche nicht im EU-Emissionshandel inkludiert sind, zu dem der Großteil der Tourismuswirtschaft gehört, liegt das Reduktionsziel bis 2030 bei $-28 \%$ gegenüber 2016. Schwerpunkte sind dabei die Emissionen in den Bereichen Verkehr und Gebäude. Beim Verkehr sollen Emissionen bis 2030 um 31,4 \% gegenüber dem aktuellen Stand gesenkt werden, bis 2050 soll schließlich die fossilfreie Mobilität erreicht werden. Im Gebäudesektor ist das Ziel bis 2030 eine Reduktion der Emissionen um 37,5 \% gegenüber dem aktuellen Stand. Bei der erneuerbaren Energie strebt Österreich aufgrund der günstigen Voraussetzungen des Landes bis 2030 einen Anteil von 45-50 \% an, deutlich über dem EU-weiten Ziel, und der Stromverbrauch soll sogar zu $100 \%$ aus erneuerbaren Quellen gedeckt werden. Im Hinblick auf die Energieeffizienz soll die Primärenergieintensität (Energieeinsatz im Verhältnis zum Bruttoinlandsprodukt) um $25-30 \%$ gegenüber 2015 gesenkt werden.

Die Strategie gliedert sich in generelle Aufgaben, deren Umsetzung weitgehend offengelassen wurde, und konkrete Leuchtturmprojekte mit Angabe von Zuständigkeiten, Instrumenten und dem jeweiligen Zeithorizont. Durch Letztere sollen die ersten Schritte zur Implementierung der Strategie gesetzt werden. Bei den Aufgaben sind für den Tourismus vor allem die Vorgaben in den Bereichen Mobilität und Gebäude von Interesse. Infrastruktur für E-Mobilität, den öffentlichen Verkehr sowie Fuß- und Radwege sollen ausgebaut werden, besonders auch im ländlichen Raum. Ausdrücklich wird dabei die Wichtigkeit des Mobilitätsmanagements im Tourismus erwähnt. Zudem soll es ein neues österreichweites Tarif- und Vertriebssystem für den öffentlichen Verkehr geben. Im Hinblick auf den Gebäudesektor wird die EU-Vorgabe befolgt, dass Neubauten nach 2020 Niedrigenergiegebäude sein müssen. Des Weiteren soll die Sanierungsrate von derzeit $1 \%$ auf $2 \%$ im Zeitraum 2020-2030 angehoben werden.

Ein weiterer Aspekt, der für touristische Unternehmen von Interesse sein wird, ist die Ankündigung eines Förderprogramms zum Energiesparmanagement bei kleinen und mittleren Unternehmen, welches auch Kosteneinsparungen und damit Wettbewerbsvorteile bringen soll. Für den Tourismus relevant sind zudem auch die Anmerkungen zu kleinskaligen Stromerzeugungsanlagen, welche aktuell bereits beispielsweise von einigen Skigebieten betrieben werden (hierzu gehört etwa die Nutzung einer Beschneiungsanlage zur Stromgewinnung außerhalb der Wintermonate und der Einsatz von Fotovoltaik an Gebäuden). Die Errichtung derartiger Anlagen soll durch Abbau von Investitionshemmnissen erleichtert werden, unter anderem durch eine Novelle des Umweltverträglichkeitsprüfungsgesetzes (UVP-G).

Auf all diese Bereiche soll auch in den Leuchtturmprojekten bereits kurzfristig eingegangen werden. Im Bereich Gebäude sollen effizientere, einfach zugängliche Programme zur Sanierungsförderung entwickelt werden, auch für Betriebsgebäude. Hinsichtlich E-Mobilität sollen die involvierten Akteure (dabei wird ausdrücklich der Tourismussektor erwähnt) über Anreize und Fördersysteme motiviert und bei der Umsetzung unterstützt werden. Beispielsweise geht es hierbei um E-Carsharing, E-Taxisysteme, bedarfsorientierte E-Mobilitätsservices, elektrische Bedarfsbusangebote, E-Bikeverleihsysteme. Zudem soll der schienengebundene Verkehr ausgebaut werden. In erster Linie betrifft dies den Nahverkehr in Ballungsräumen, es sollen aber auch neue Nachtzugverbindungen eingerichtet werden, um die Zahl der Kurzstreckenflüge zu verringern. $\mathrm{Zu}$ den einzelnen „Leuchttürmen“ werden jeweils auch die zuständigen Verwaltungseinheiten (Ministerien, Länder etc.) genannt sowie auch mögliche Förderinstrumente und ein grober Zeithorizont zur Umsetzung. Genauere Angaben zu Durchführung und Finanzierung der einzelnen Maßnahmen gibt es jedoch nicht.

Auf Grundlage der Klima- und Energiestrategie wurde der Nationale Energie- und Klimaplan (NEKP) erstellt, der bis Ende 2019 an die Europäische Kommission zu übermitteln ist. Der erste Entwurf erntete viel Kritik, u. a. von wissenschaftlicher Seite, und wurde auch von der Europäischen Kommission zurückgewiesen. Auf Initiative des Vertreters der Wissenschaft im Nationalen Klimaschutzkomitee Gottfried Kirchengast (Uni Graz, ÖAW), Helga Kromp-Kolb (BOKU), Karl Steininger (Uni Graz) und Sigrid Stagl (WU) wurde ein Referenzplan als Grundlage für einen wissenschaftlich fundierten und mit den Pariser Klimazielen in Einklang stehenden Nationalen Energie- und Klimaplan für Österreich (Ref-NEKP) erstellt (Kirchengast et al. 2019). Den Tourismus (indirekt) betreffend hervorzuheben sind dabei Forderungen nach einer Besteuerung des Flugverkehrs, der Ausbau des internationalen Bahnverkehrs und der Nachtreisezüge sowie die Entwicklung eines verlässlichen Buchungssystems für internationale Bahnreisen. Während beispielweise der Ausbau der Nachtreisezüge aufgenommen wurde, sind eine weitere Ökologisierung des Anreiz-, Förder- und Steuersystems oder die Ausweitung des Handelssystems (ETS) auf weitere Sektoren auch im finalen Plan, der im Dezember 2019 vorgelegt wurde, 
nur als optionale Maßnahmen enthalten. Die nun inkludierte Wirkungsfolgenabschätzung zeigt auch auf, dass mit den enthaltenen Maßnahmen das Treibhausgasreduktionsziel von $-36 \%$ bis 2030 nicht erreicht wird.

Bereits ein Jahr vor der Klima- und Energiestrategie 2018 beschloss die österreichische Bundesregierung im Jahr 2017 auch eine Klimawandelanpassungsstrategie (BMNT 2017a, b). Wird der Tourismus in Ersterer nur am Rande erwähnt wird, spielt er in der Anpassungsstrategie eine zentrale Rolle, als eines von 14 Aktivitätsfeldern. Allgemeine Zielsetzung ist dabei die „Sicherung Österreichs als attraktiver und nachhaltiger Tourismusstandort durch Nutzung klimawandelbedingter Potenziale und Forcierung umweltfreundlicher Anpassungsmaßnahmen“. Als Maßnahmen werden folgende Punkte vorgeschlagen:

- Berücksichtigung des Klimawandels in den Tourismusstrategien,

- Entwicklung von klimaschonenden Anpassungsmaßnahmen auf Basis der Tourismusstrategien,

- Ausarbeitung, Bereitstellung und Verbesserung regionaler Daten als Entscheidungsgrundlage für Anpassungsmaßnahmen,

- Unterstützung klimawandelgefährdeter Wintersportregionen bei der Schaffung von schneeunabhängigen Angeboten,

- Stärkung des alpinen Sommertourismus,

- Ausbau des Städtetourismus in Österreich.

Zudem beinhaltet die Strategie auch Querverbindungen anderer Aktionsfelder zum Tourismus, etwa im Hinblick auf Raumordnung (Förderung einer klimawandelangepassten, nachhaltigen touristischen Infrastruktur) und Wasserwirtschaft (betreffend künstliche Beschneiung).

Mit Ausnahme des Burgenlandes verfügen auch die österreichischen Bundesländer über eigene Klimaschutz- und/oder Anpassungsstrategien beziehungsweise integrierte Strategien, welche beide Aspekte berücksichtigen (Magistrat der Stadt Wien 2009; Amt der OÖ. Landesregierung 2013; Amt der Tiroler Landesregierung 2015; Amt der Vorarlberger Landesregierung 2015; Land Salzburg 2015, 2017; Amt der NÖ Landesregierung 2017a; Amt der Steiermärkischen Landesregierung 2017a, b; Land Kärnten 2018; Umweltbundesamt 2018). Im Hinblick auf den Klimaschutz gehen diese bislang u. a. auf die Förderung der Anreise per Bahn (Tirol, Vorarlberg) und eines emissionsarmen Mobilitätsangebots in Wien und in den Tourismusregionen (Vorarlberg, Niederösterreich, Steiermark), auf Energieraumplanung touristischer Destinationen (Tirol), umweltfreundliche Events (Tirol, Steiermark), den Ausbau von Informationsangeboten und Marketingmaßnahmen für klimaschonende Urlaube in Österreich (Steiermark) und die Forcierung des österreichischen Umweltzeichens für Tourismusbetriebe (Wien) ein.
Bezüglich der Klimawandelanpassung wird u. a. der Ausbau des Ganzjahrestourismus (Vorarlberg, Salzburg, Steiermark), des Städtetourismus (Steiermark) sowie wetter- und schneeunabhängiger Angebote (Kärnten), der Schutz der Infrastruktur für den Alpintourismus (Steiermark), eine verstärkte Bewusstseinsbildung zu durch den Klimawandel verursachten Gefahren im alpinen Raum (Tirol), die Erhebung relevanter Daten für den Tourismus (Tirol) sowie die Bereitstellung regionaler Klimaszenarien als Entscheidungsgrundlage (Steiermark) angeführt.

Dabei beschränken sich einige der Bundesländerstrategiepapiere auf ein einfaches Aufzählen möglicher Maßnahmen, während in anderen versucht wird, die Umsetzung möglichst genau zu planen, mit Definition der verantwortlichen Akteure, des zeitlichen Horizonts, einer Abschätzung der Kosten, u. Ä. Besonders vorbildlich - auch im Vergleich mit der österreichischen Klima- und Energiestrategie - sind hier die Klimastrategie Tirol und die Klimawandelanpassungsstrategie der Steiermark.

\subsubsection{Berücksichtigung des Klimawandels in den österreichischen Tourismusstrategien}

Wie in der österreichischen Klimawandelanpassungsstrategie gefordert, sollte der Klimawandel auch in den Tourismusstrategien entsprechend berücksichtigt werden. In der österreichischen Tourismusstrategie von 2010 (BMWFJ 2010) wurde dieses Thema noch lediglich am Rande erwähnt. In den Tourismusstrategien der Bundesländer (Land Vorarlberg 2012; Land Salzburg 2013; Wien Tourismus 2014; Land Kärnten 2016; Land Steiermark 2016; Land Tirol et al. 2016; Amt der NÖ Landesregierung 2017b; Amt der OÖ. Landeregierung et al. 2018; Burgenland Tourismus GmbH 2018), welche in den letzten Jahren veröffentlicht wurden, spielen die Klimawandelanpassung und der Klimaschutz in manchen Fällen bereits eine wichtige Rolle, während andere, wie im Falle der Strategien von Kärnten und Burgenland, den Klimawandel nicht erwähnen. Auch in der niederösterreichischen Strategie kommt der Klimaschutz nicht vor, die Klimawandelanpassung wird hier aber zumindest nebenbei erwähnt. Ohne Anmerkungen zur Klimawandelanpassung kommen auch die Tourismusstrategien von Oberösterreich, Wien und Vorarlberg aus, wenngleich in der zuvor erwähnten Vorarlberger Klimawandelanpassungsstrategie interpretiert wird, dass die Maßnahmen der Tourismusstrategie des Landes indirekt durchaus auf eine Stärkung des Ganzjahrestourismus abzielen. Als vorbildlich kann die Salzburger Strategie gelten, welche sowohl zu Klimaschutz als auch zur Anpassung die Angabe konkreter Maßnahmen beinhaltet. Im Bereich Klimaschutz sind auch die Vorarlberger und die Wiener Strategie hervorzuheben, welche der nachhaltigen Mobilität eine zentrale Position einräumen. 
In der Salzburger Strategie wird im Hinblick auf Anpassung in erster Linie eine Fortführung der bereits 2005 in der Tourismuspolitik des Landes aufgestellten Zielsetzung genannt, welche das Land als Ganzjahresdestination positionieren soll. Vermehrt soll dabei auf Kongress-, Gesundheits-/Wellness- und Kulturtourismus sowie Sportveranstaltungen gesetzt werden. Im Wintertourismus sollen schneeunabhängige Angebote wie Winterwanderwege und die Themen Advent und Stille Nacht ausgebaut werden. Die Maßnahmen betreffend Klimaschutz laufen unter der Zielvorgabe, dass das Land Salzburg ein Reiseziel mit einem grünen Image sein sollte. Hierzu zählen Vorgaben im Bereich klimaschonende Anreise (z. B. Förderung der E-Mobilität in Tourismusregionen und -orten), Einsatz regionaler Lebensmittel in der Gastronomie, ökologische Baumaßnahmen im Tourismus sowie Bewusstseinsbildung und Schulung touristischer Anbieter zu den Themen Nachhaltigkeit und Energieeffizienz.

In der Vorarlberger Strategie sind zwei der sechs übergeordneten Ziele für den Klimaschutz relevant, erstens die Positionierung des Landes als Modellregion für umweltfreundliche Mobilität im Tourismus (u. a. durch eine Zusammenarbeit aus Verkehrsverbund und Vorarlberg Tourismus zur Steuerung des Mobilitätsverhaltens der Gäste) und zweitens die Mitarbeit des Tourismussektors an der Zielsetzung des Landes, bis 2050 Energieautonomie zu erreichen. Dafür sollen Maßnahmen gesetzt werden, um die Energieeffizienz zu erhöhen und die Nutzung erneuerbarer Energieträger auszubauen.

Wien schließlich soll zur Smart Tourism City werden, als ein internationales Beispiel für nachhaltiges Mobilitätsmanagement im Städtetourismus. Prioritäten sind dabei die komfortable Anbindung des Flughafens an den öffentlichen Verkehr, digitales Mobilitätsmanagement, Ausweitung des E-Taxibetriebs sowie spezielle Mobilitätsangebote und Dienstleistungen für Touristinnen und Touristen wie mehrsprachige Infopoints an zentralen Verkehrsknotenpunkten und eine Mobilitätskarte, welche die Nutzung von öffentlichen Verkehrsmitteln, Citybikes, Garagen und Carsharing ermöglicht.

Im Frühjahr 2019 wurde schließlich auch auf nationaler Ebene eine neue Strategie vorgestellt, der „Plan T“, welcher auf Basis eines partizipativen Prozesses entwickelt wurde (BMNT 2019). Eines der neun prioritären Handlungsfelder dieser Strategie trägt den Titel „Lebensgrundlage nachhaltig sichern“. Das Hauptaugenmerk liegt dabei auf der Erhöhung der Energie- und Ressourceneffizienz, dem Engagement touristischer Betriebe in der Erzeugung erneuerbarer Energie und dem Ausbau nachhaltiger Mobilitäts- und Verkehrslösungen. Im Aktionsplan 2019/2020 zur Umsetzung des „Plan T“ wird in diesem Zusammenhang die Entwicklung von Tourismusregionen zu Klima- und Energiemodellregionen und die Einbeziehung von touristischen Betrieben und Destinationen in erneuerbare Energiegemeinschaften genannt, unter Nutzung von Fördergeldern aus dem 100.000-Dächer-Fotovoltaik- und Kleinspeicherprogramm. Abgesehen vom Verweis auf diese Förderprogramme werden keine weiteren Details zur Umsetzung und Finanzierung der vorgesehenen Maßnahmen genannt. Zur Klimawandelanpassung bemerkt der „Plan T“ nur, es ,... muss das touristische Angebot an die veränderten Rahmenbedingungen angepasst werden“ (BMNT 2019: 26). Genauere Ausführungen, wie dieses Ziel erreicht werden sollte, beinhaltet der Plan nicht.

\subsection{Treibhausgasreduktionsmaßnahmen für den Tourismus in der gesamten Wertschöpfungskette}

Die Erläuterungen in Kap. 11 zeigen, dass die An- und Abreise der wichtigste Emissionsaspekt ist, da insbesondere der Flugverkehr aufgrund der zurückgelegten Entfernungen und hohen Energieintensität schnell die Gesamtemissionen stark ansteigen lässt. Sobald dieses Verkehrsmittel involviert ist, werden andere Maßnahmen in der Wertschöpfungskette etwa eine besonders $\mathrm{CO}_{2}$-arme Beherbergung - vergleichsweise bedeutungslos. Auch die Anreise mit Privat-Pkw hat einen um ein Vielfaches höheren $\mathrm{CO}_{2}$-Fußabdruck als mit öffentlichen Verkehrsmitteln (die Elektrifizierung der PkwFlotte schreitet dafür noch zu langsam voran und kann aufgrund der noch unvollständigen Dekarbonisierung des eingesetzten Stroms keine ausreichend positiven Auswirkungen auf die Gesamtemissionen erzielen). Sowohl Friesenbichler (2003) als auch Unger et al. (2016) empfehlen daher Destinationen, ihren Gästen verstärkt die Anreise mit dem Zug oder Bus nahezulegen und dazu entsprechende Angebote zu machen (Anbindung an Skigebiete, nahtloser Gepäcktransport zur Destination, Abholung am Bahnhof).

Aus einer systemischen Perspektive sollte das Ziel sein, einen möglichst hohen Anteil von Anreisen mit Bus oder Bahn zu erreichen. Dazu wäre es notwendig, Nahmärkte auszubauen (Gössling et al. 2015) und vor Ort die Mobilität der Gäste durch den Nahverkehr, Mobilitätsservicedienstleistungen, Carsharingangebote und Formen der geteilten Mikromobilität (Räder, E-Scooter) sicherzustellen. Um den Anteil der Transportemissionen an den Gesamtemissionen niedrig zu halten, ist auch die durchschnittliche Aufenthaltslänge von zentraler Bedeutung. In Österreich ist beispielsweise die durchschnittliche Aufenthaltsdauer von 4,9 Nächten im Jahr 1990 auf 3,3 Nächte im Jahr 2018 gesunken, also um 32 \% (Statistik Austria 2019). Daraus ergibt sich, dass eine immer größere Zahl von Gästen notwendig ist, um die Zahl der Übernachtungen im Land auf dem gleichen Niveau zu halten, und damit vergrößert sich potenziell immer auch der Anteil der Emissionen aus An- und Abreise im Verhältnis zu den Gesamtemissionen.

Abb. 12.1 verdeutlicht die Bedeutung der durchschnittlichen Aufenthaltsdauer im Vergleich zur Anreisedistanz. Auffallend ist, dass insbesondere Gäste aus den Niederlan- 
Abb. 12.1 Aufenthaltsdauer und Anreisedistanz der Besucher Österreichs (2015). (Basierend auf Statistik Austria 2017; Nachdruck aus Gössling et al. 2019 mit Genehmigung von Taylor \& Francis Ltd)

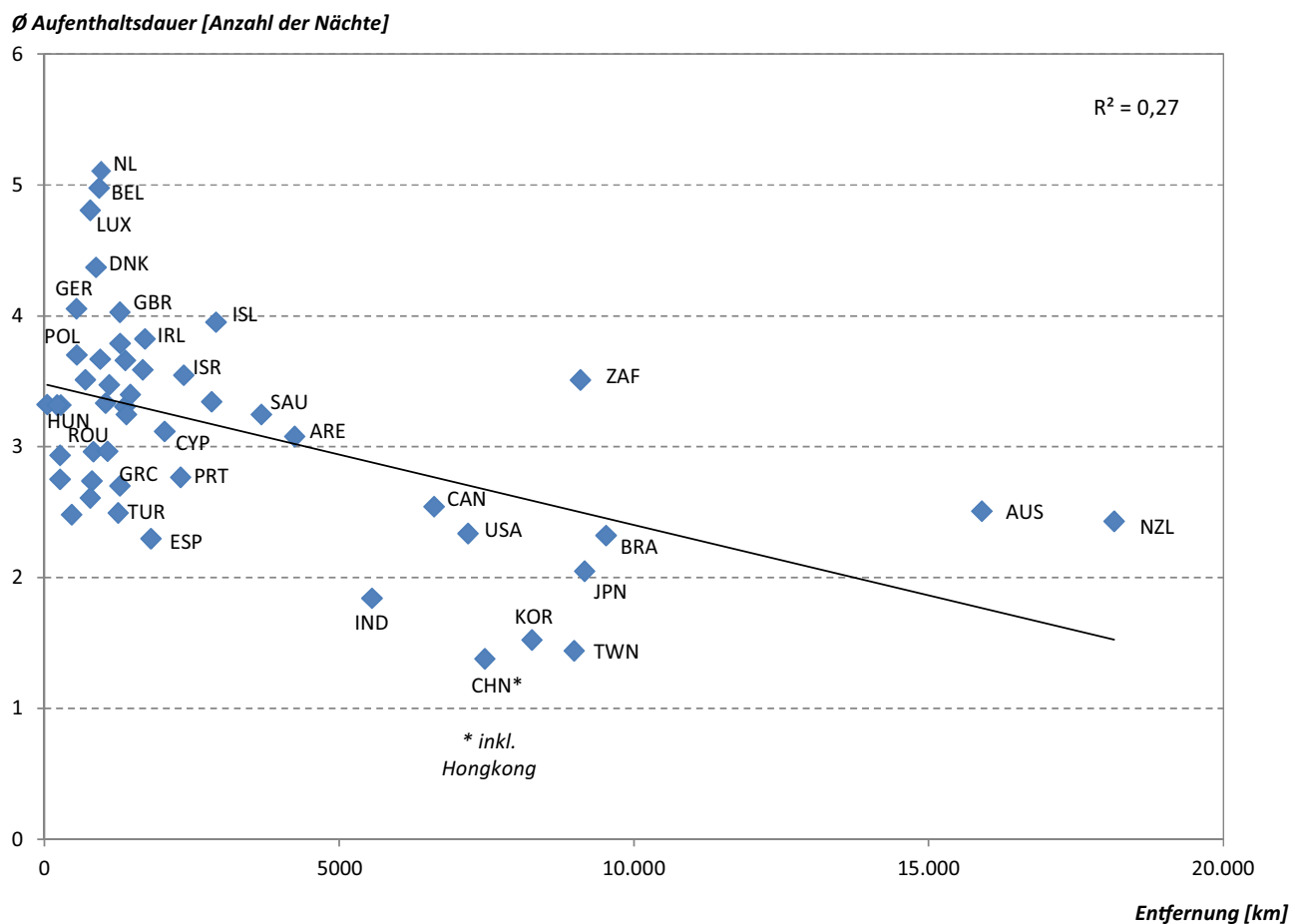

den, Luxemburg, Belgien, Dänemark und Deutschland eine höhere Aufenthaltsdauer haben als die besonders weit gereisten Gäste aus z. B. China oder Japan mit häufig komplexen Besuchsprogrammen in mehreren europäischen Ländern. Die Entwicklung von Nahmärkten kann aus diesem Grund zu einer höheren durchschnittlichen Aufenthaltsdauer beitragen und gleichzeitig Transportemissionen verringern.

Mittelfristig wäre es auch notwendig, marktbasierte Maßnahmen für den Flugverkehr einzuführen, wie beispielsweise auch von der internationalen Zivilluftfahrtorganisation gefordert (ICAO 2015), die das Interesse an einem hohen Belegungsgrad der Maschinen und der Verwendung alternativer Brennstoffe erhöhen. Es ist zum Beispiel denkbar, nichtbelegte Sitze zu besteuern und gleichzeitig eine Grundabgabe (Air Passenger Duty) einzuführen, um zu verhindern, dass Sitze sehr billig verkauft werden. Diese Abgabe könnte sich an der britischen Flugsteuer orientieren, die distanzbasiert gestaffelt und damit näherungsweise proportional zu den verursachten Emissionen ist. Gleichzeitig wäre es notwendig, der Erforschung der technischen Möglichkeiten eines synthetischen Brennstoffs bzw. einer hybridelektrischen Antriebsform verstärkt Aufmerksamkeit zu widmen, also den Antrieben, die langfristig tatsächlich emissionsfrei sein könnten. Steigende Treibstoffkosten für den Flugverkehr sind ein wichtiger Baustein, um diesen Antriebsformen zur Markteinführung zu verhelfen (Gössling und Scott 2018).

Weitere systemische Maßnahmen sollten insbesondere die Gastronomie betreffen. Lenzen et al. (2018) haben die Nahrungsmittelproduktion, deren Verarbeitung und Zubereitung als zweitwichtigsten Aspekt der Gesamtemissionen her- vorgehoben. Die großen Möglichkeiten der Gastronomie, in diesem Bereich Emissionsminderungen zu erreichen, sind in Kap. 5 ausgeführt. Die Ergebnisse legen den Schluss nahe, dass ein wesentlicher Teil der Gesamtemissionen allein durch geringfügige Änderungen der Einkaufs-, Koch- und Präsentationsstrategien eingespart werden könnten. Einsparungen in der gleichen Größenordnung wären auch in der Beherbergung allein durch die Umstellung auf Strom aus erneuerbaren Quellen möglich, wobei in diesem Bereich das grundsätzliche Potenzial einer fast klimaneutralen Wirtschaftsweise gegeben ist (Gössling 2010).

Einzelmaßnahmen können ebenfalls zu signifikanten Treibhausgaseinsparungen führen. Zum Beispiel zeigen die Analysen von Pröbstl und Jiricka (2012a) für die Skilifte Lech (Vorarlberg) ein hohes Einsparpotenzial beim Pistenmanagement. Insgesamt $70 \%$ des Dieselverbrauchs entfallen auf die Pistenpräparation, wo es, ebenso wie beim Schneemanagement, erhebliche Einsparpotenziale gibt (z. B. durch GPS-basierte Schneehöhenmessung). Auch bei den Skiliften gilt, dass durch die Verwendung regenerativ erzeugten Stroms signifikante Emissionsminderungen erreicht werden können (Pröbstl und Jiricka 2012b).

Österreich kann auf verschiedene Best-Practice-Ansätze verweisen, die international viel Beachtung gefunden haben. ${ }^{1}$ Allen gemeinsam ist, dass sie „Klimafreundlichkeit“ zum Alleinstellungsmerkmal gemacht haben und sich dadurch

1 Dazu gehören zum Beispiel das Wiener Boutiquehotel Stadthalle (www.hotelstadthalle.at), die Biohotels (www.biohotels.info) sowie das Mobilitätskonzept der Alpinen Perlen (www.alpine-pearls-com). 
neue Märkte erschließen konnten. Obwohl die Konzepte zum Teil mit öffentlichen Mitteln gefördert wurden, können zentrale Einsichten aus der Entwicklung und dem Betrieb auch in der Fläche umgesetzt werden. Klimaschonendes Wirtschaften kann zu Kosteneinsparungen verhelfen, Gast- und Mitarbeiterloyalität erhöhen und Impulse für die Destinationsentwicklung geben. Staatliche und regionale finanzielle Anreize könnten ein Interesse an diesen Konzepten zusätzlich verstärken. Hierzu gehören auch Zertifizierungen, wie das Österreichische Umweltzeichen, oder ein nach EMAS bzw. ISO 14001 zertifiziertes Umweltmanagementsystem (Pröbstl und Müller 2012; Reschl 2019).

Allerdings ist bei der Buchung für die Kunden vielfach die Berücksichtigung nachhaltiger Entwicklungen nur eingeschränkt möglich. Wichtig wären in diesem Zusammenhang Partnerschaften mit Reiseveranstaltern, Reisebüros und Buchungsplattformen, wie z. B. TUI, Booking.com oder trivago.

Es gibt viele weitere internationale Best-Practice-Beispiele, die aus einer Treibhausgasminderungsperspektive für Österreich interessant sein könnten. Dazu gehören z. B. Angebote der Klimakompensation, da nicht für alle Gäste eine klimaschonende Anreise infrage kommt. Dies ist teilweise von Veranstaltern im deutschen Forum Anders Reisen eingeführt worden. Auch vor Ort können viele Angebote gemacht werden, um Mobilität nachhaltiger zu gestalten. Dazu gehören einerseits Apps, die es Gästen möglich machen, lokale und regionale Mobilitätsangebote lückenlos zu nutzen und dabei auch verschiedene Transportmittel zu kombinieren (z. B. WienMobil). Eine wünschenswerte Entwicklung aus touristischer Sicht wäre eine zentrale App, die innerhalb ganz Österreichs genutzt werden kann, z. B. nach dem Vorbild von Moovel $^{2}$. Auch die Förderung der ÖPNV-Nutzung sei hier erwähnt, mit Gratisnutzungsangeboten durch Gästekarten (z. B. KONUS-Gästekarte im Schwarzwald, Schladming-Dachstein Sommercard, AchenseeCard, Geneva Transport Card für Hotelgäste). Weiters lassen sich durch die Bereitschaft bei der Förderung der Elektromobilität durch die Hotelbetriebe weitere Problemstellungen realisieren (Last Mile, Auftanken/ Aufladen usw.; vgl. Abschn. 4.4.3).

Insbesondere in Städten können Fahrrad- und Fußverkehr gefördert werden. Hotels könnten ihren Gästen zum Beispiel als Standard ein kostenloses Fahrrad anbieten. ${ }^{3}$ Da auch Nutzer anderer Unterkunftsformen potenziell am Fahrradverleih interessiert sind, bietet sich außerdem ein Mietangebot in Bahnhofsnähe an. ${ }^{4}$ Fußverkehr kann auch durch einfachste Mittel gefördert werden. Die Stadt Pontevedra in Spanien

\footnotetext{
2 www.moovel-group.com.

3 Im Ohboy-Hotel in Malmö (www.ohboy.se) gehört zu jedem Zimmer ein Fahrrad.

4 In den Niederlanden bietet OV-Fiets (www.ns.nl) zum Preis von 3,85€ pro 24 Stunden Räder in fast 300 Leihstationen an, die mit minimalem Aufwand gemietet werden können.
}

hat beispielsweise eine entsprechende Innenstadtkarte entwickelt, die Entfernungen in Metern und Gehminuten ausweist (Abb. 12.2). Auch Wanderwege bzw. Sehenswürdigkeiten lassen sich so den Reisenden näherbringen.

Durch autonome Mobilität werden sich bald neue Möglichkeiten des Transportes ergeben. Gäste, die mit dem Zug anreisen, können beispielsweise von selbstfahrenden Fahrzeugen an ihr Ziel gebracht werden. Die Wechselwirkungen dieser neuen Technologien sind aber noch weitgehend ungeklärt.

\subsection{Die Bedeutung klimaverträglicher Lebensstile für den Tourismus}

Aus einer gesamtgesellschaftlichen Perspektive erstreckt sich das Erfordernis einer drastischen und systematischen Emissionsreduktion naheliegend über alle gesellschaftlichen Gruppen und über alle Branchen. Mit Blick auf das Handeln einzelner Menschen sind die individuellen Aktivitäten in sämtlichen Lebensbereichen klimapolitisch relevant. Gleichzeitig ist die verbleibende Zeit, um das Wirtschaftssystem im Sinne der Pariser Klimaziele zu ,dekarbonisieren“, also den Ausstoß von $\mathrm{CO}_{2}$ und anderen Treibhausgasen $\mathrm{zu}$ minimieren, äußerst knapp. Diese Zeitknappheit wird insbesondere dann deutlich, wenn man berücksichtigt, dass eine Dekarbonisierung ,,von heute auf morgen“" weder gesamtwirtschaftlich noch in Bezug auf die individuellen Lebensrealitäten der Menschen realistisch ist. Die Einhaltung der Pariser Klimaziele unter Berücksichtigung ökonomischer, sozialer, politischer und technologischer Realitäten kann jedoch nur gelingen, wenn die dringend notwendige Reduktion von Treibhausgasemissionen zu einem Projekt für alle wird, das alle relevanten Zielgruppen berücksichtigt und einen „Paris Lifestyle“ zu einer attraktiven Art der Lebensgestaltung werden lässt (Schwarzinger et al. 2018). Das Spektrum von klimaverträglichen Produkten und Dienstleistungen, die sowohl für die Angebots- als auch für die Nachfrageseite attraktiv sind, mag aus heutiger Perspektive klein erscheinen und wie eine Schnittmenge wirken, die sich auf Nischenmärkte beschränkt. Allerdings steht außer Frage, dass es erforderlich ist, diese Schnittmenge systematisch zu vergrößern, um das Wirtschaftssystem, von dem auch der Tourismus ein wesentlicher Bestandteil ist, auf einen klimaverträglichen Kurs zu bringen. Wege zu einem klimaverträglichen touristischen Produkt können auch ohne eine entsprechende Kennzeichnung angestrebt werden und auch unbemerkt vom Gast implementiert werden.

Die Tatsache, dass Angebote mit betont geringen Klimaauswirkungen - speziell im Tourismus - derzeit noch eine Ausnahme darstellen, darf also nicht zur Annahme verleiten, dass derartige Angebote auch zukünftig ihren Nischencharakter behalten werden. Vorweg sei an dieser Stelle angemerkt, 


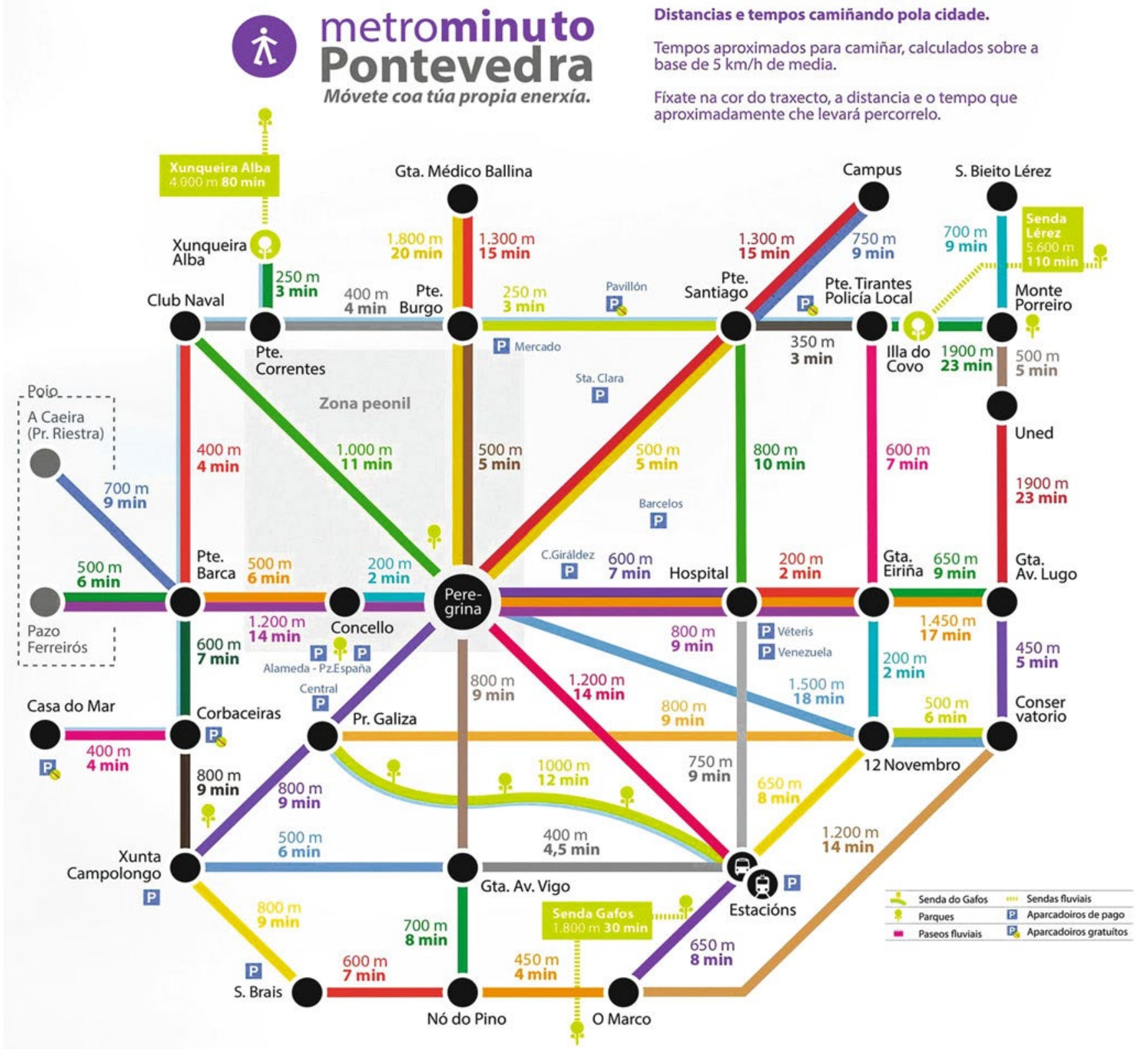

Abb. 12.2 Beispiel zur Förderung des Fußverkehrs - Karte der Entfernungen für Fußgänger in Pontevedra, Spanien. (Concello de Pontevedra 2016)

dass heute klimaschädliche Angebote nicht zwangsläufig von der Bildfläche verschwinden müssen. Die Dekarbonisierung der Tourismuswirtschaft dreht sich stark um die Frage, wie das Portfolio einer Region klimaverträglich gemacht werden kann. In manchen Fällen, man denke beispielsweise an den Umstieg auf Strom aus erneuerbaren Energieträgern, gestaltet sich die Dekarbonisierung relativ unproblematisch. In anderen Fällen, etwa im Verkehr, sind die Hürden auf dem Weg zur Klimaverträglichkeit wesentlich größer. Oftmals ist derzeit also noch offen, welche aktuell klimaschädlichen Angebote in Zukunft durch technologische Entwicklungen klimaneutral werden können und in welchen Fällen eine Ver- schiebung der Nachfrage durch regulatorische Eingriffe unausweichlich ist. Die derzeit in Bezug auf den Klimaschutz wenig zufriedenstellende Situation im Tourismus ist primär dem Umstand geschuldet, dass es aktuell für die Branche noch kaum verbindliche Vorgaben für eine sukzessive Emissionsreduktion gibt. Bei einer konsumbasierten Emissionsbilanz von in Österreich rund 15 Tonnen $\mathrm{CO}_{2}$-Äquivalent pro Kopf und Jahr (Windsperger et al. 2017) und einem Zielwert von nahe null im Jahr 2050, ergibt sich ein Reduktionspfad entsprechend Abb. 12.3, der aus heutiger Sicht ebenso ambitioniert wie alternativlos ist. Um das Pariser Klimaziel einer Beschränkung der globalen Erwärmung von zumindest weni- 
Abb. 12.3 Idealisierter Reduktionspfad bis 2050. Konsumbasierte Emissionen pro Kopf in Österreich für das Jahr 2015. (Gemäß Windsperger et al. 2017; Grafik: Prettentahler, Gössling, Damm und Neger 2020)

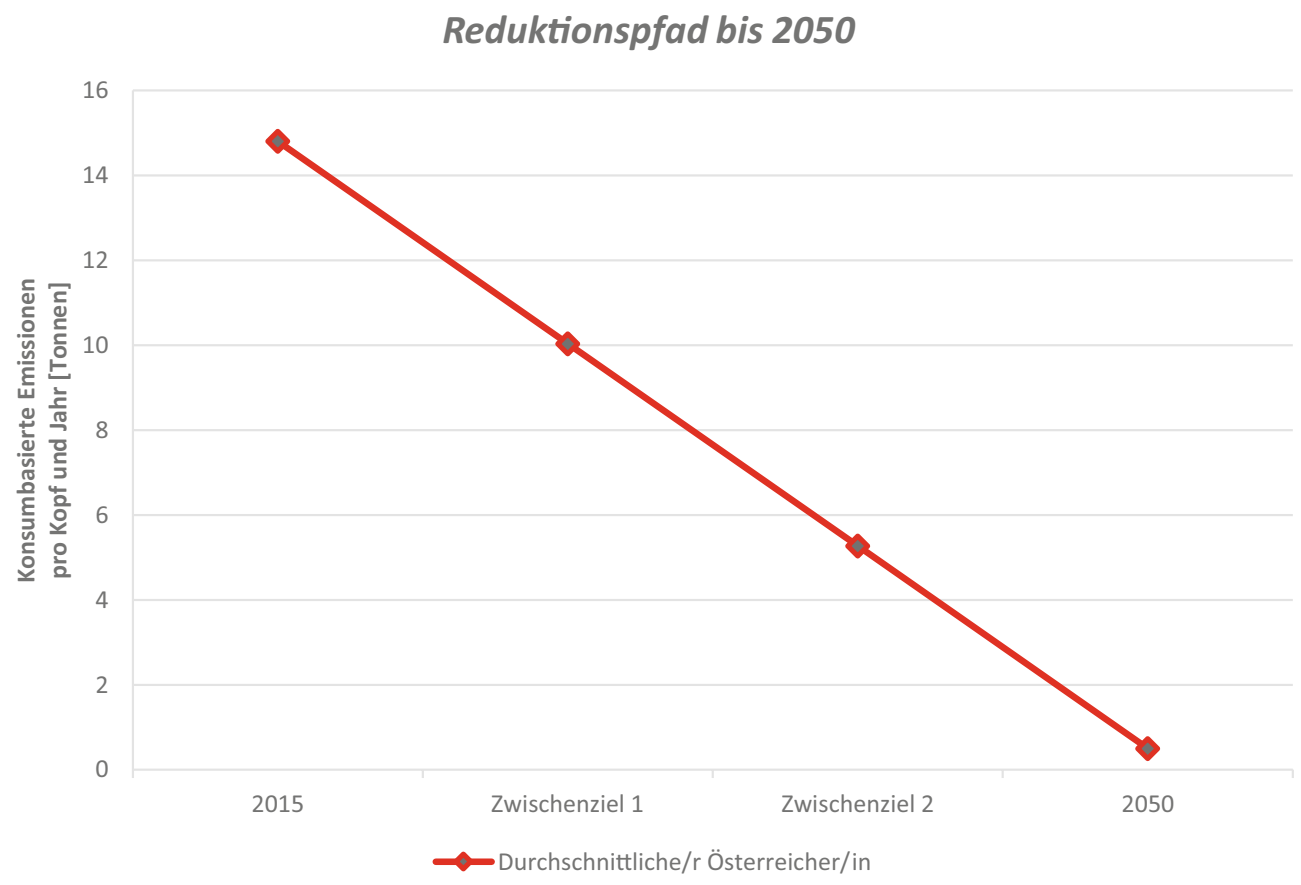

ger als $2{ }^{\circ} \mathrm{C}$ im Vergleich zum vorindustriellen Zeitalter einzuhalten, sind also ohne Zweifel weitreichende Maßnahmen erforderlich. Darunter fallen neben Lenkungsmaßnahmen wie einer ökologischen Steuerreform mit dem mittelfristigen Ziel einer vollständigen Internalisierung externer Kosten (d. h. die Zurechnung der externen (Umwelt-)Effekte auf den Verursacher) insbesondere die entsprechenden Reaktionen der Anbieter, um in einem nach Klimaschutzaspekten optimierten Wirtschaftssystem noch immer ein wirtschaftlich tragfähiges Angebot sicherzustellen. Nimmt man zwischen dem Basisjahr 2018 und dem Zieljahr 2050 vereinfacht einen linearen Reduktionspfad an, so wird deutlich, dass der zu beschreitende Pfad durch das gegebene Treibhausgasrestbudget ${ }^{5}$ stei- $^{-}$ ler wird, wenn erst später entsprechende Maßnahmen gesetzt werden.

Was für die erforderliche Emissionsreduktion auf Individualebene der Fall ist, gilt natürlich auch für Anbieter von all jenen Produkten und Dienstleistungen, die sich auf die Emissionsbilanz der Konsumenten auswirken. Für Wirtschaftsbetriebe ergibt sich aus dieser prozessualen Perspektive gemäß Abb. 12.3 eine besondere Situation: Je später wirksame Maßnahmen zur Emissionsreduktion gesetzt werden, desto größer wird zu einem späteren Zeitpunkt der Aufwand sein, der vonnöten ist, um die Bereitstellung des eigenen Angebots doch noch auf einen klimaverträglichen Weg zu bringen. Ein späterer Reduktionsbeginn bei gleichbleibendem Restbudget erfordert einen steileren Pfad und ein früheres „Null“-Niveau. Werden also emissionsreduzierende Maßnahmen zugunsten

\footnotetext{
5 Jene Menge an Treibhausgasen, die weltweit noch höchstens freigesetzt werden darf, um das $2^{\circ} \mathrm{C}$-Ziel erreichen zu können.
}

kurzfristiger wirtschaftlicher Überlegungen hinausgezögert, vergrößert dies den zu einem späteren Zeitpunkt nötigen Aufwand dramatisch. Allerdings kann es auch sein, dass die Kosten für diese Reduktion zu einem späteren Zeitpunkt geringer sind. Diese Unsicherheit kann durch über die Zeit degressive Förderungen insofern reduziert werden, als dadurch die Preise von zielführenden Maßnahmen heute und in Zukunft angeglichen werden und somit kein wirtschaftlicher Anreiz zur Verschiebung der Investition mehr besteht.

Die prozessuale Zieldefinition mit einer sukzessiven Entwicklung in Richtung Energiewende ist aber nicht nur aus unmittelbar betriebswirtschaftlicher Sicht relevant, sondern auch im Hinblick auf die Frage, wie Menschen zukünftig ihre Konsum- und Verhaltensentscheidungen treffen werden. Aktuelle Befunde aus der empirischen Forschung sind sich weitgehend einig darüber, dass das klima- und umweltspezifische Problembewusstsein in westlichen Gesellschaften relativ gut ausgeprägt ist. In einer Studie des deutschen Umweltbundesamts gaben beispielsweise $88 \%$ der Befragten an, das Ziel der Treibhausgasneutralität für sehr wichtig (52\%) oder eher wichtig $(36 \%)$ zu halten. $91 \%$ halten es für erforderlich, Wirtschaft und Märkte so zu regulieren, dass Umweltbelastungen gering gehalten werden. Zudem zeigt sich „,in großen Teilen der Gesellschaft eine Offenheit für Veränderungen, auch mit Blick auf die Umsetzung einer nachhaltigeren Wirtschafts- und Lebensweise“ sowie ein breiter Rückhalt „für ein noch stärkeres umweltpolitisches Engagement in Wirtschaft, Politik und Gesellschaft" (BMUB und UBA 2016, S. 23).

Zusätzlich legen internationale Daten einen signifikanten Zusammenhang zwischen Wirtschaftswachstum und Umweltbewusstsein nahe (Franzen 2003; Franzen und Vogl 
2013). Ein klares Bekenntnis zum Klimaschutz und die Bereitstellung von klimaverträglichen Angeboten fallen also besonders bei Bürgerinnen und Bürgern aus jenen Ländern auf fruchtbaren Boden, in denen die Wirtschaftslage gut ist. Urlaubern aus wirtschaftlich prosperierenden Ländern kann also ein tendenziell höheres Umweltbewusstsein attestiert werden, wenn damit derzeit auch noch nicht unbedingt ein tatsächlich geringerer Ressourcenbedarf assoziiert ist (Schwarzinger et al. 2019a). Dies unterstreicht nicht nur die Relevanz dieser Zielgruppen für die klima- und energiebezogene Maßnahmenentwicklung, sondern macht die Bereitstellung klima- und umweltverträglicher Angebote für ebendiese Zielgruppen zum Gegenstand kurzfristigerer wirtschaftlicher Überlegungen.

Allerdings stellt sich nach wie vor die Frage, warum ein ausgeprägtes Umweltbewusstsein nicht immer in ökologisch orientiertem Verhalten resultiert (Newton und Meyer 2013; Aune et al. 2016; Binder und Blankenberg 2017; Arnold et al. 2018). Diese Diskrepanz zwischen Werthaltung und Handeln wird üblicherweise als Attitude-Action Gap bezeichnet (Newton und Meyer 2013). Relativ klein ist diese Diskrepanz bei Entscheidungen und Verhaltensweisen, die mit einem eher geringen subjektiven Aufwand einhergehen, wie es beispielsweise bei Mülltrennen oder dem Einsatz von LED-Leuchtmitteln im Haushalt der Fall ist. Groß ist die Diskrepanz zwischen Werthaltung und eigenem Verhalten hingegen vor allem dann, wenn der subjektiv empfundene Nachteil oder Komfortverlust groß ist. Der Verzicht auf eine lange angestrebte Fernreise oder der Verzicht auf das Auto zum Preis einer längeren Wegzeit sind hierfür gute Beispiele. Dieser Unterschied zwischen Entscheidungen mit einem unterschiedlich hohen persönlichen Einsatz wurde in der sogenannten Low-cost-Hypothese ausführlich behandelt (Diekmann und Preisendörfer 2003). Umwelt- und klimabewusstes Handeln stößt also oft dann an seine Grenzen, wenn es den persönlichen Komfort oder die Verwirklichung von Wünschen zu gefährden scheint. Stehen klimabewusste Konsumentinnen und Konsumenten vor der Wahl zwischen zwei touristischen Angeboten mit subjektiv vergleichbarem Nutzen sowie gleichen Kosten und eines der beiden Angebote weist eine wesentlich schlechtere Klimabilanz auf, so gibt es keine naheliegende Veranlassung, die emissionsmäßig schädlichere Alternative zu wählen. Die gegenwärtig schlechte Verfügbarkeit von Informationen über die Klimabilanz von Produkten und Dienstleistungen führt allerdings dazu, dass selbst motivierte und handlungsbereite Personen kaum in der Lage sind, eine informierte Konsumentscheidung zu treffen (Schwarzinger et al. 2019b). Daraus ergeben sich zwei Ansatzpunkte:

1) Über die Treibhausgasbilanz von Produkten und Dienstleistungen muss Transparenz hergestellt werden, um eine faire Grundlage für die Internalisierung externer Kosten zu schaffen und um klimabewussten Konsumentinnen und
Konsumenten eine informierte Entscheidung im Einklang mit ihren ökologischen Werthaltungen sowie mit ihrem Lebensstil und Geschmack zu ermöglichen (Bourdieu 1987).

2) Darüber hinaus sind Maßnahmen bzw. touristische Angebote zu entwickeln, bei denen auch nichtklimabewusste Konsumentinnen und Konsumenten die Erwartungen an ihren Aufenthalt (Komfort, Erlebniskomponente etc.) erfüllt sehen, während sie (quasi unbemerkt) klimaschonend konsumieren (vgl. Kap. 5, 13).

\subsection{Handlungsoptionen, Kommunikations- und Forschungsbedarf}

Österreich hat das Pariser Klimaabkommen ratifiziert und die österreichische Politik ist daher gefordert, die nötigen Rahmenbedingungen - nicht nur für den Tourismus - zu schaffen, um die ambitionierten Treibhausgasemissionsvorgaben auf europäischer Ebene zu erreichen. Neben allgemeinen Zielvorgaben braucht es auch die Verpflichtung zur Umsetzung konkreter Maßnahmen. Der Energie- und Klimaplan und die Klimawandelanpassungsstrategie auf nationaler Ebene liefern dazu einige erste Ansätze, bleiben aber vielfach in ihrer Formulierung noch sehr offen. So ist im finalen Klima- und Energieplan eine weitere Ökologisierung des Anreiz-, Förderund Steuersystems nur als „optional“ formuliert. Es gibt hier angesichts der mangelnden Reduktionszielerreichung insgesamt noch Nachbesserungsbedarf. Auch im Hinblick auf den Tourismus fehlen konkretere Maßnahmen und Umsetzungspläne, vor allem im Verkehrs- und Steuerbereich. Auf Ebene der Bundesländer würden auch eine stärkere Zusammenarbeit und Vereinheitlichung der Strategien sowie Anpassung an nationale Vorgaben eine Möglichkeit bieten, die derzeit noch sehr unterschiedlich ausgeprägten Bemühungen im Klimaschutz und in der Klimawandelanpassung im Tourismus anzugleichen. Dabei ist insbesondere der Fernreiseverkehr zu berücksichtigen, der auch laut Peeters (2017) einer der wichtigsten Ansatzpunkte zur Erreichung eines klimaneutralen Tourismus ist. Jedoch auch der hohe Autoanreiseanteil im nationalen Tourismus ist zu beachten. Die Anreise per Bahn und Bus und die autofreie Mobilität vor Ort sind daher möglichst einfach und günstig zu gestalten. ${ }^{6}$ Andererseits wäre es im Sinne des Klimaschutzes sinnvoll, wenn sich der heimische Tourismus wieder stärker auf nahe liegende Märkte fokussieren würde. Ein möglicher Beitrag der Politik wäre es, Forschung zu energieeffizientem Transport bestmöglich zu unterstützen und durch Besteuerung emissionsstarke Trans-

\footnotetext{
${ }^{6}$ Eine Ausweitung des von der im Januar 2020 angelobten Bundesregierung angekündigten Österreich-Tickets (Nutzung der öffentlichen Verkehrsmittel für $3 €$ pro Tag für ganz Österreich bzw. $1 €$ pro Tag je Bundesland) auch für die touristische Nutzung (z. B. als Wochenticket) wäre hier ein Meilenstein.
} 
portweisen weniger attraktiv zu machen, insbesondere den Flugverkehr. An dieser Stelle sei auch die Rolle der Fluglinien erwähnt und deren Möglichkeit, Emissionsreduktionen im Flugverkehr deutlich proaktiver voranzutreiben und entsprechende Maßnahmen umzusetzen, z. B. die Förderung von synthetischen Treibstoffen, Weiterentwicklung neuer Antriebssysteme (z. B. Hybridantriebe), eine Optimierung der Flugrouten, oder zumindest Kompensationsprojekte zu forcieren.

Klima- und Energiestrategien auf unterschiedlichen Ebenen können nur dann einen Beitrag leisten, wenn sie auch tatsächlich umgesetzt werden. Eine kontinuierliche Evaluierung der nationalen Strategien wäre hier dienlich, um aufzuzeigen, wo noch Aufholbedarf besteht. Zudem ist es sinnvoll, den Umsetzungsgrad der bisherigen Strategien in diesem Bereich sowie deren Auswirkungen zu untersuchen, um festzustellen, wo Schwierigkeiten und Hindernisse aufgetreten sind, um diese in Zukunft gezielt zu vermeiden und eine effizientere Implementierung zu erreichen. Welche Rahmenbedingungen notwendig sind, um die Akzeptanz klimaschonender Angebote zu erhöhen, sind in weiterführender Forschung zu ermitteln.

Die bestehenden und in Zukunft möglicherweise verstärkten Vorgaben auf der Makroebene sowie auch auf der Mikroebene, im Hinblick auf das steigende Umweltbewusstsein der Konsumenten, stellen die Tourismuswirtschaft vor strategische Herausforderungen. Es wird sowohl an den Destinationen als auch an den Anbietern liegen, ein entsprechend emissionsreduziertes Tourismusangebot zu schaffen, das die klima- und umweltbewussten Konsumenten - ihren Werthaltungen und objektiven Erfordernissen folgend - konsumieren können. Im Sinne der Nudge-Theorie (Thaler und Sunstein 2008) können Produkte entwickelt werden, die den Gast unbemerkt beeinflussen und eventuell sogar eher als zusätzlicher Service empfunden werden. Ein Nudge verändert das Verhalten der Menschen auf vorhersehbare Weise, ohne Optionen zu verbieten oder ihre wirtschaftlichen Anreize wesentlich zu ändern (Thaler und Sunstein 2008). In Anbetracht dieser Faktoren, durch die konventionelle Angebote tendenziell unter Druck geraten, ist es ratsam, sich in der Branche so früh wie möglich mit der Dekarbonisierung ihrer wirtschaftlichen Prozesse sowie mit der psychologischen Wirkung emissionsreduzierender Maßnahmen zu befassen und diese zur Umsetzung zu bringen.

Klimaverträgliche Lebensstile können zwar eine Rolle in der Emissionsreduktion im Tourismus spielen und stellen damit ein Potenzial dar, das auf jeden Fall in der Klimapolitik so gut wie möglich genutzt und, etwa durch Bewusstseinsbildung, gefördert werden kann. Um jedoch tatsächlich einen $\mathrm{CO}_{2}$-neutralen Tourismus in Österreich zu erreichen, werden Verhaltensänderungen, die nur von einem Teil der Konsumentinnen und Konsumenten getragen werden - und auch hier oft wieder nur unter optimalen Voraussetzungen -, nicht ausreichen. Will man die Pariser Klimaziele erreichen, ist es daher notwendig, dass die Politik aktiv regulierend eingreift, um die heimische Tourismuswirtschaft in eine klimaschonende Richtung zu lenken, und auch die Unternehmen brauchen noch einen proaktiveren und systematischeren $\mathrm{Zu}$ gang zu einer entsprechenden Angebotsentwicklung.

\subsection{Zusammenfassung}

Die wichtigsten Maßnahmen zur Reduktion der Treibhausgase im Tourismus betreffen den Transport (hohe Übereinstimmung, starke Beweislage). Dabei ist es besonders wichtig, auf Nahmärkte zu fokussieren, dem Trend zu einer geringen Aufenthaltsdauer entgegenzuwirken sowie attraktive öffentliche Verkehrsangebote in Bezug auf Anreise und den Nahverkehr in den Destinationen zu schaffen. Der Flugverkehr muss dagegen unattraktiver werden, wenn die Zahl der Passagiere verringert werden soll. Passagiere und Politik haben es auch in der Hand, Fluggesellschaften zu stärkeren $\mathrm{CO}_{2}$-Reduktionsmaßnahmen zu bewegen (vgl. auch Ausführungen in Kap. 13).

Ein weiterer Bereich, wo hohe Einsparungen erzielt werden können, ist die Gastronomie, besonders beim Einkauf von regionalen und ökologisch erzeugten Produkten (vgl. Kap. 5). Weiters sollte die Energie aus erneuerbaren Quellen bezogen werden. In der Beherbergung ist damit sogar die Möglichkeit gegeben, fast völlige $\mathrm{CO}_{2}$-Neutralität zu erreichen (vgl. Kap. 4). Einsparungspotenziale gibt es auch bei touristischen Outdooraktivitäten, in Bezug auf den alpinen Wintertourismus sind dabei beispielsweise ein optimiertes Pistenmanagement und der Einsatz von Ökostrom vielversprechend. Best-Practice-Beispiele aus dem In- und Ausland können bei der Umsetzung all dieser Maßnahmen den Weg weisen. Sie zeugen außerdem davon, dass vielfach neben den verringerten Emissionen auch andere positive Effekte erzielt werden können, wie Kosteneinsparungen in der Energienutzung.

Als Fortführung der seit den 1990er-Jahren begonnenen Bestrebungen zu einer internationalen Klimapolitik wurde 2015 das als historisch geltende Pariser Klimaabkommen vereinbart, in welchem sich der Großteil der Länder der Welt zu einer Reduktion der Treibhausgasemissionen verpflichtet hat, welche es möglich machen soll, dass der globale Temperaturanstieg unterhalb von $2{ }^{\circ} \mathrm{C}$ gegenüber dem vorindustriellen Niveau bleibt. Die EU und ihre Mitgliedsstaaten sind geschlossen am Pariser Abkommen beteiligt, mit der ambitionierten Vorgabe, die Treibhausgasemissionen um $40 \%$ im Vergleich zu 1990 zu reduzieren. Dazu hat die Europäische Kommission verschiedene Maßnahmen gesetzt, wobei allerdings der Tourismus kaum Erwähnung findet. Indirekt ist die Tourismuswirtschaft aber sehr wohl durch Vorgaben etwa in den Bereichen Energieeffizienz von Gebäuden oder emissionsfreie Mobilität betroffen. Auch in der europäischen Klima- 
wandelanpassungsstrategie ist der Tourismus kein prioritäres Thema. In der Tourismuspolitik der Europäischen Kommission wird dagegen großer Wert auf ökologische Nachhaltigkeit - bei der die Reduktion der Treibhausgasemissionen beinhaltet ist - gelegt. Die wichtigsten Aktionen dafür sind die Förderung der Entwicklung nachhaltiger transnationaler Tourismusprodukte, die Erstellung eines Indikatorensystems, das Destinationen dabei unterstützen soll, sich nachhaltig zu entwickeln, und die Unterstützung von Nachhaltigkeit auf betrieblicher Ebene durch Beratung und die Möglichkeit der Zertifizierung mit dem EU Ecolabel für umweltfreundliche Unternehmen.

Die 2018 vorgestellte österreichische Klima- und Energiestrategie und der darauf aufbauende nationale Energie- und Klimaplan (NEKP) beinhalten allgemeine Vorschläge und Maßnahmen, die - zumeist indirekt - auch wieder den Tourismus betreffen, besonders in den Bereichen Mobilitätsmanagement, öffentlicher Verkehr, E-Mobilität, Energiesparen und die Erzeugung erneuerbarer Energien in kleinskaligen Anlagen. In der österreichischen Klimawandelanpassungsstrategie aus dem Jahr 2017 wird der Tourismus als eines der zentralen Handlungsfelder berücksichtigt. Dabei werden vor allem die Notwendigkeit einer ausreichenden Datenverfügbarkeit, das Angebot schneeunabhängiger Alternativen in Wintersportdestinationen sowie die Erstellung von Querverbindungen zu anderen Bereichen wie Raumordnung und Wasserwirtschaft betont. Als Alternative zu den zu erwartenden Verlusten im Winter wird auf eine stärkere Forcierung des Sommer- und Städtetourismus gesetzt. In dem 2019 vorgestellten „Plan T“, welcher eine Strategie für die touristische Entwicklung auf nationaler Ebene darstellt, wird die Klimawandelanpassung hingegen nicht erwähnt, dafür ist Nachhaltigkeit aber ein zentrales Ziel. Dabei geht es insbesondere wiederum hauptsächlich um Energieeffizienz, erneuerbare Energien und möglichst emissionsarme Mobilität.

In den österreichischen Bundesländern gibt es Klimastrategien, in denen teilweise der Tourismus berücksichtigt wird, sowie Tourismusstrategien, die mitunter auch die Aspekte Klimaschutz und Klimawandelanpassung beinhalten. Dabei gibt es von Bundesland zu Bundesland große Unterschiede. Während diesen Themen in einigen Strategien eine hohe Bedeutung zugemessen wird, werden sie in anderen nur am Rande erwähnt oder völlig ignoriert.

Um auch auf individueller Ebene zum Erreichen der Ziele des Pariser Klimaabkommens beitragen zu können, kann der „Paris Lifestyle“ propagiert werden - ein Lebensstil, bei dem Treibhausgasemissionen so gut wie möglich vermieden werden sollten. Angesichts der konsumbasiert ermittelten Emissionsbilanz der Österreicher mit $15 \mathrm{t} \mathrm{CO}_{2}$-Äquivalenten pro Kopf (2015), weit entfernt vom Zielwert von nahe null im Jahr 2050, erfordert dies drastische Änderungen, auch im Reiseverhalten (hohe Übereinstimmung, mittlere Beweislage). Hier stellt sich momentan noch das Problem, dass es wenige klimaschonende Angebote gibt oder dass diese um ein Vielfaches teurer sind, sodass auch Klimabewusste oft zu weniger umweltfreundlichen Alternativen greifen (hohe Übereinstimmung, mittlere Beweislage).

\section{Kernaussagen - Kapitel 12}

- In verschiedenen Bereichen des Tourismussektors (Beherbergung, Gastronomie, touristische Aktivitäten und insbesondere Transport) kann durch entsprechende Maßnahmen eine signifikante Emissionsreduktion erreicht werden (hohe Übereinstimmung, mittlere Beweislage).

- In den Strategien zur Reduktion der Treibhausgasemissionen der Europäischen Kommission und der österreichischen Bundesregierung ist der Tourismus kein prioritäres Thema (hohe Übereinstimmung, schwache Beweislage).

- Die Tourismusstrategien auf europäischer, nationaler und Länderebene beinhalten teilweise Maßnahmen zur Emissionsreduktion, aber kaum konkrete Vorgehensweisen zu Finanzierung, Priorisierung und Zeitplänen (hohe Übereinstimmung, schwache Beweislage).

- Um die Ziele des Pariser Klimaabkommens zu erreichen, sind ein möglichst emissionsarmer Lebensstil („Paris Lifestyle“) inklusive klimaschonenden Reisens und die Schaffung entsprechender Angebote alternativlos (hohe Übereinstimmung, mittlere Beweislage).

- Um das Pariser Klimaziel einer Beschränkung der globalen Erwärmung von zumindest weniger als $2{ }^{\circ} \mathrm{C}$ im Vergleich zum vorindustriellen Zeitalter einzuhalten, sind weitreichende Maßnahmen erforderlich, die durch die österreichische Klimapolitik bislang nicht ausreichend initiiert, gefördert und umgesetzt werden (hohe Übereinstimmung, schwache Beweislage).

\section{Literatur}

Amt der NÖ Landesregierung (2017a) Niederösterreichisches Klimaund Energieprogramm 2020. 2. Auflage, Amt der Niederösterreichischen Landesregierung, St. Pölten, Österreich. Online unter: http:// www.noe.gv.at/noe/Klima/KlimaEnergieprogramm2020.html (letzter Zugriff: 13.05.2020).

Amt der NÖ Landesregierung (2017b) Tourismusstrategie Niederösterreich 2020. Amt der Niederösterreichischen Landesregierung, St. Pölten, Österreich. Online unter: http://www.noe.gv.at/noe/Wirtschaft-Tourismus-Technologie/17_NOE_Tourismusstrategie2020 low_144.pdf (letzter Zugriff: 13.05.2020).

Amt der OÖ. Landesregierung (2013) Ö̈. Klimawandel-Anpassungsstrategie. Amt der Oberösterreichischen Landesregierung, Linz, Österreich. Online unter: https://www.land-oberoesterreich.gv.at/ files/publikationen/us_klimawandelanpass.pdf (letzter Zugriff: 13.05.2020). 
Amt der OÖ. Landesregierung, Wirtschaftskammer Oberösterreich \& Oberösterreich Tourismus GmbH (2018) Tourismusstrategie 2022: Tourismus, Zukunft, Oberösterreich. Amt der Oberösterreichischen Landesregierung, Wirtschaftskammer Oberösterreich und Oberösterreich Tourismus GmbH, Linz, Österreich. Online unter: https://www. oberoesterreich-tourismus.at/fileadmin/user_upload/oberoesterreichtourismus/Bilder/B2B/LTO/Dokumente/Oberoesterreich_LandesTourismusstrategie_2022_web.pdf (letzter Zugriff: 13.05.2020).

Amt der Steiermärkischen Landesregierung (2017a) Klima- und Energiestrategie Steiermark 2030. Amt der Steiermärkischen Landesregierung, Graz, Österreich. Online unter: https://www.technik. steiermark.at/cms/ziel/142705670/DE/ (letzter Zugriff: 13.05.2020).

Amt der Steiermärkischen Landesregierung (2017b) Klimawandelanpassung-Strategie Steiermark 2050. Amt der Steiermärkischen Landesregierung, Graz, Österreich. Online unter: https://www.technik. steiermark.at/cms/ziel/102834231/DE/ (letzter Zugriff: 13.05.2020).

Amt der Tiroler Landesregierung (2015) Klimastrategie Tirol. Amt der Tiroler Landesregierung, Innsbruck, Österreich. Online unter: https:// www.tirol.gv.at/landesentwicklung/nachhaltigkeit/klimaschutz/tiroler-klimastrategie/ (letzter Zugriff: 13.05.2020).

Amt der Vorarlberger Landesregierung (2015) Strategie zur Anpassung an den Klimawandel in Vorarlberg: Ziele, Herausforderungen und Handlungsfelder. Amt der Vorarlberger Landesregierung, Bregenz, Österreich. Online unter: https://vorarlberg. at/documents/21336/122370/AdVL+2016_Strategie+zur+Anpassung+an+den+Klimawandel_web.pdf/22c-̄1 afa-40ad-4074-a8e93beb9bba2d4f (letzter Zugriff: 13.05.2020).

Arnold, O., Kibbe, A., Hartig, T. \& Kaiser, F.G. (2018) Capturing the environmental impact of individual lifestyles: evidence of the criterion validity of the general ecological behavior scale. Environment and Behavior 50(3), 350-372. DOI: https://doi. org/10.1177/0013916517701796

Aune, M., Godbolt, Å.L., Sørensen, K.H., Ryghaug, M., Karlstrøm, H. \& Næss, R. (2016) Concerned consumption: global warming changing household domestication of energy. Energy Policy 98, 290-297. DOI: https://doi.org/10.1016/j.enpol.2016.09.001

Binder, M. \& Blankenberg, A.-K. (2017) Green lifestyles and subjective well-being: more about self-image than actual behavior? Journal of Economic Behavior \& Organization 137, 304-323. DOI: https://doi. org/10.1016/j.jebo.2017.03.009

BMNT (2017a) Die österreichische Strategie zur Anpassung an den Klimawandel Teil 1 - Kontext. Bundesministerium für Nachhaltigkeit und Tourismus (BMNT), Wien, Österreich. Online unter: https:// www.bmlrt.gv.at/umwelt/klimaschutz/klimapolitik national/anpassungsstrategie/strategie-kontext.html (letzter Zugriff: 13.05.2020).

BMNT (2017b) Die österreichische Strategie zur Anpassung an den Klimawandel Teil 2 -Aktionsplan. Bundesministerium für Nachhaltigkeit und Tourismus (BMNT), Wien, Österreich. Online unter: https:// www.bmlrt.gv.at/umwelt/klimaschutz/klimapolitik_national/anpassungsstrategie/strategie-kontext.html (letzter Zugriff: 13.05.2020).

BMNT (2019) Plan T - Masterplan für Tourismus. Bundesministerium für Nachhaltigkeit und Tourismus (BMNT), Wien, Österreich. Online unter: https://www.bmnt.gv.at/tourismus/masterplan_tourismus. html (letzter Zugriff: 07.05.2019).

BMNT \& BMVIT (2018) \#mission2030. Die österreichische Klimaund Energiestrategie. Bundesministerium für Nachhaltigkeit und Tourismus (BMNT) und Bundesministerium für Verkehr, Innovation und Technologie (BMVIT), Wien, Österreich. Online unter: https:// www.bmlrt.gv.at/service/publikationen/umwelt/mission-2030oesterreichische-klima-und-energiestrategie.html (letzter Zugriff: 13.05.2020)

BMUB \& UBA (2016) Umweltbewusstsein in Deutschland 2016: Ergebnisse einer repräsentativen Bevölkerungsumfrage. Bundesministerium für Umwelt, Naturschutz, Bau und Reaktorsicherheit (BMUB), Berlin und Umweltbundesamt (UBA), Dessau-Roßlau, Deutschland. Online unter: https://www.umweltbundesamt.de/sites/ default/files/medien/376/publikationen/umweltbewusstsein_deutschland 2016 bf.pdf (letzter Zugriff: 13.05.2020).

BMWFJ (2010) Neue Wege im Tourismus: die neue österreichische Tourismusstrategie. Bundesministerium für Wirtschaft, Familie und Jugend (BMWFJ), Wien, Österreich. Online unter: https://www. bmlrt.gv.at/dam/jcr:7d0389dd-4ae8-4726-b254-b88b90da00f0/ Strategie_Neue\%20Wege\%20im\%20Tourismus.pdf (letzter Zugriff: 13.05.2020).

Bourdieu, P. (1987) Distinction: a social critique of the judgement of taste. Harvard University Press, Cambridge, MA, USA.

Burgenland Tourismus GmbH (2018) Tourismusstrategie Burgenland $2022+$. Burgenland Tourismus GmbH, Eisenstadt, Österreich. Online unter: https://www.burgenland.info/xstorage/1/PDFs/Tourismusstrategie-Burgenland-2022-.pdf (letzter Zugriff: 13.05.2020).

Concello de Pontevedra (2016) Metrominuto. Concello de Pontevedra, Pontevedra, Spanien. Online unter: http://www.pontevedra.gal/ publicacions/Metrominuto/esp/files/assets/common/downloads/ publication.pdf (letzter Zugriff: 04.02.2020).

Diekmann, A. \& Preisendörfer, P. (2003) Green and greenback: the behavioral effects of environmental attitudes in low-cost and high-cost situations. Rationality and Society 15(4), 441-472. DOI: https://doi. org/10.1177/1043463103154002

Europäische Kommission (2013) The EU strategy on adaptation to climate change. Europäische Kommission, Brüssel, Belgien. Online unter: https://ec.europa.eu/clima/policies/adaptation/what en\#tab-0-1 (letzter Zugriff: 04.08.2018).

Europäische Kommission (2016a) Paris Agreement to enter into force as EU agrees ratification. Europäische Kommission, Brüssel, Belgien. Online unter: https://ec.europa.eu/clima/news/articles/ news_2016100401_en (letzter Zugriff: 04.08.2018).

Europäische Kommission (2016b) Eine europäische Strategie für emissionsarme Mobilität. Europäische Kommission, Brüssel, Belgien. Online unter: https://ec.europa.eu/transparency/regdoc/rep/1/2016/ DE/1-2016-501-DE-F1-1.PDF (letzter Zugriff: 04.08.2018).

Europäische Kommission (2016c) The European Tourism Indicator System: ETIS toolkit for sustainable destination management. Europäische Union, Luxemburg. Online unter: https://ec.europa.eu/ docsroom/documents/21749 (letzter Zugriff: 10.05.2019).

Europäische Kommission (2018) Clean energy for all Europeans. Europäische Kommission, Brüssel, Belgien. Online unter: https:// ec.europa.eu/energy/en/topics/energy-strategy-and-energy-union/ clean-energy-all-europeans (letzter Zugriff: 06.09.2018).

Europäische Kommission (2019a) Tourism. Europäische Kommission, Brüssel, Belgien. Online unter: https://ec.europa.eu/growth/sectors/ tourism_en (letzter Zugriff: 10.05.2019).

Europäische Kommission (2019b) The EU ecolabel scheme. Europäische Kommission, Brüssel, Belgien. Online unter: http://ec.europa. eu/environment/ecolabel/the-ecolabel-scheme.html (letzter Zugriff: 10.05.2019).

Europäisches Parlament \& Rat der Europäischen Union (2010) Richtlinie 2010/31/EU des Europäischen Parlaments und des Rates vom 19. Mai 2010 über die Gesamtenergieeffizienz von Gebäuden. Amtsblatt der Europäischen Union. Online unter: https://eur-lex.europa. eu/legal-content/EN/ALL/;ELX_SESSIONID=FZMjThLLzfxmmM CQGp2Y1s2d3TjwtD8QS3pqdkhXZbwqGwlgY9KN!2064651424? uri=CELEX\%3A32010L0031 (letzter Zugriff: 06.09.2018).

Franzen, A. (2003) Environmental attitudes in international comparison: an analysis of the ISSP surveys 1993 and 2000. Social Science Quarterly 85(2), 297-308. DOI: https://doi.org/10.1111/15406237.8402005

Franzen, A. \& Vogl, D. (2013) Two decades of measuring environmental attitudes: a comparative analysis of 33 countries. Global Environmental Change 23(5), 1001-1008. DOI: https://doi.org/10.1016/j. gloenvcha.2013.03.009 
Friesenbichler, J. (2003) Energieeinsatz und $\mathrm{CO}_{2}$-Emissionen im Wintertourismus. Diplomarbeit im Rahmen des Fachhochschulstudiengangs „Infrastrukturwirtschaft“ der FH Joanneum Kapfenberg, Österreich.

Gössling, S. (2010) Carbon management in tourism. Routledge, London, Vereinigtes Königreich.

Gössling, S. \& Scott, D. (2018) The decarbonisation impasse: global tourism leaders' views on climate change mitigation. Journal of Sustainable Tourism 26(12), 2071-2086. DOI: https://doi.org/10.1 080/09669582.2018.1529770

Gössling, S., Scott, D. \& Hall, C.M. (2015) Inter-market variability in $\mathrm{CO}_{2}$ emission-intensities in tourism: implications for destination marketing and carbon management. Tourism Management 46, 203 212. DOI: https://doi.org/10.1016/j.tourman.2014.06.021

Gössling, S., Scott, D. \& Hall, C.M. (2019) Global trends in length of stay: implications for destination management and climate change. Journal of Sustainable Tourism 26(12), 2087-2101. DOI: https://doi. org/10.1080/09669582.2018.1529771

ICAO (2015) An introduction to market-based measures. International Civil Aviation Organization (ICAO). Online unter: https://www. icao.int/Meetings/EnvironmentalWorkshops/Documents/2015Warsaw/6_1_An-introduction-to-market-based-measures-MBMs. pdf (letzter Zugriff: 23.05.2019).

Kirchengast, G., Kromp-Kolb, H., Steininger, K., Stagl, S., Kirchner, M., Ambach, Ch., Grohs, J., Gutsohn, A., Peisker, J. \& Strunk, B. (2019) Referenzplan als Grundlage für einen wissenschaftlich fundierten und mit den Pariser Klimazielen in Einklang stehenden Nationalen Energie- und Klimaplan für Österreich (Ref-NEKP). Gesamtband, November 2019, CCCA Wien-Graz. Verlag der Österreichischen Akademie der Wissenschaften (ÖAW), Wien, Österreich. Online unter: https://ccca.ac.at/fileadmin/00_DokumenteHauptmenue/03_ Aktivitaeten/UniNEtZ_SDG13/RefNEKP/Ref-NEKP_Gesamtband_ Nov2019_VerlOeAW.pdf (letzter Zugriff: 13.05.2020).

Land Kärnten (2016) Aussichten: Tourismusstrategie des Landes Kärnten. Land Kärnten, Klagenfurt, Österreich. Online unter: https:// www.ktn.gv.at/DE/repos/files/ktn.gv.at/Abteilungen/Abt7/importneu/export1385_inuse/Strategische_Wirtschaftsentwicklung_und Tourismus/Kaerntner_Tourismusstrategie.pdf?exp=116630\&fps $=-$ 9d267d7ac3bcbfde11d51f752608bb211476553f (letzter Zugriff: 13.05.2020).

Land Kärnten (2018) Klimastrategie Kärnten. Entwurf, Stand Jänner 2018. Land Kärnten, Klagenfurt, Österreich. Online unter: https:// ccca.ac.at/fileadmin/00_DokumenteHauptmenue/08_Newsletter/ klimastrategie_ges_red_180426.pdf (letzter Zugriff: 13.05.2020).

Land Salzburg (2013) Salzburger Tourismus: Gesund. Innovativ. Nachhaltig. Strategieplan Tourismus 2020. Land Salzburg, Salzburg, Österreich. Online unter: https://www.salzburg.gv.at/wirtschaft_ Documents/Publikationen\%20Wirtschaft-Tourismus/strategieplantourismus2020.pdf (letzter Zugriff: 13.05.2020).

Land Salzburg (2015) Masterplan Klima + Energie 2020im Rahmen der Klima- und Energiestrategie SALZBURG 2050. Land Salzburg, Salzburg, Österreich. Online unter: https://www.salzburg.gv.at/umweltnaturwasser_/Documents/1s_16_klima-folder_web.pdf (letzter Zugriff: 13.05 .2020$)$.

Land Salzburg (2017) Strategie zur Anpassung an den Klimawandel in Salzburg im Rahmen der Klima- und Energiestrategie SALZBURG 2050. Land Salzburg, Salzburg, Österreich. Online unter: https:// www.salzburg.gv.at/umweltnaturwasser_Documents/Strategie $\% 20$ zur\%20Anpassung\%20an\%20den\%20Klimawandel\%20in $\% 20$ Salzburg_WEB-V24-05-18.pdf (letzter Zugriff: 13.05.2020).

Land Steiermark (2016) Tourismusstrategie Steiermark 2025: Wachstum durch Qualität. Land Steiermark, Graz, Österreich. Online unter: https://www.verwaltung.steiermark.at/cms/dokumente/ 11721185_74836018/27da75e5/Tourismusstrategie $\% 202025 \% 20$ ohne $\% 20$ Vorwort.pdf (letzter Zugriff: 13.05.2020).

Land Tirol, Tirol Werbung, Wirtschaftskammer Tirol \& Verband der Tiroler Tourismusverbände (2016) Der Tiroler Weg 2021: Kernbot- schaft einer Strategie für den Tiroler Tourismus. Land Tirol, Tirol Werbung, Wirtschaftskammer Tirol \& Verband der Tiroler Tourismusverbände, Innsbruck, Österreich. Online unter: https://www.tirolwerbung.at/wp-content/uploads/2016/06/strategie-tiroler-weg-2021. pdf (letzter Zugriff: 13.05.2020).

Land Vorarlberg (2012) Tourismusstrategie 2020: Der gemeinsame Weg in die touristische Zukunft auf Vorarlberger Art. Land Vorarlberg, Bregenz, Österreich. Online unter: http://tourismus2020.at/tourismusstrategie/ (letzte Zugriff: 13.05.2020).

Lenzen, M., Sun, Y.Y., Faturay, F., Ting, Y.P., Geschke, A. \& Malik, A. (2018) The carbon footprint of global tourism. Nature Climate Change 8(6), 522-528. DOI: https://doi.org/10.1038/s41558-0180141-x

Lettische EU-Ratspräsidentschaft \& Europäische Kommission (2015) Intended nationally determined contribution of the EU and its member states. Europäische Union. Online unter: https://ec.europa.eu/ clima/sites/clima/files/docs/2015030601_eu_indc_en.pdf (letzter Zugriff: 13.05.2020).

Magistrat der Stadt Wien (2009) Klimaschutzprogramm der Stadt Wien, Fortschreibung 2010-2020. Magistrat der Stadt Wien, Wien. Online unter: https://www.wien.gv.at/umwelt/klimaschutz/pdf/klip2-lang. pdf (letzter Zugriff: 13.05.2020).

Newton, P. \& Meyer, D. (2013) Exploring the attitudes-action gap in household resource consumption: does "environmental lifestyle" segmentation align with consumer behaviour? Sustainability 5(3), 1211-1233. DOI: https://doi.org/10.3390/su5031211

Peeters, P.M. (2017) Tourism's impact on climate change and its mitigation challenges: how can tourism become "climatically sustainable"? Dissertation, Delft University of Technology, Niederlande. DOI: https://doi.org/10.4233/uuid:615ac06e-d389-4c6c-810e$7 \mathrm{a} 4 \mathrm{ab} 5818 \mathrm{e} 8 \mathrm{~d}$

Pröbstl, U. \& Jiricka, A. (2012a) Carbon foot print Skilifte Lech. Modellprojekt in Zusammenarbeit mit dem OITAF Umweltausschuss. ISR Internationale Seilbahn-Rundschau 4/2012, 24-25. Online unter: https://de.isr.at/fileadmin/isr.at/Media/Heft-Archiv/2012/ISR2012-4.pdf (letzter Zugriff: 13.05.2020).

Pröbstl, U. \& Jiricka, A. (2012b) Sommer am Berg - mit oder ohne erneuerbare Energieträger? ISR Internationale Seilbahn Rundschau 5/2012, 23-25. Online unter: https://de.isr.at/fileadmin/isr.at/Media/ Heft-Archiv/2012/ISR-2012-5.pdf (letzter Zugriff: 13.05.2020).

Pröbstl, U. \& Müller, F. (2012) Hotel certification and its relevance for sustainable development: examples from the European Alps. In: Pineda, F.D. \& Brebbia, C.A. (Hrsg.) Sustainable Tourism V, S. 3-15. WIT Press, Ashurst, Vereinigtes Königreich. DOI: https:// doi.org/10.2495/ST120011

Reschl, J. (2019) Umweltkennzeichnungsprogramme im Kontext nachhaltigen Konsums: eine Analyse am Beispiel des Österreichischen Umweltzeichens für Tourismusbetriebe. Masterarbeit am Institut für Landschaftsentwicklung, Erholungs- und Naturschutzplanung (ILEN), Universität für Bodenkultur Wien, Österreich.

Schleussner, C.F., Rogelj, J., Schaeffer, M., Lissner, T., Licker, R., Fischer, E.M., Knutti, R., Levermann, K.F., Frieler, K. \& Hare, W. (2016) Science and policy characteristics of the Paris Agreement temperature goal. Nature Climate Change 6(9), 827-835. DOI: https://doi.org/10.1038/nclimate3096

Schwarzinger, S., Bird, D.N. \& Hadler, M. (2018) The "Paris Lifestyle" - bridging the gap between science and communication by analysing and quantifying the role of target groups for climate change mitigation and adaptation: an interdisciplinary approach. In: Leal Filho, W., Lackner, B. \& McGhie, H. (Hrsg.) Addressing the challenges in communicating climate change across various audiences, S. 375397. Springer, Cham, Schweiz. DOI: https://doi.org/10.1007/9783-319-98294-6_23

Schwarzinger, S., Bird, D.N., Lettmayer, G., Henriksen, I.M., Skjolsvold, T.M., Olaeta, X.U., Alvarez, L.P., Velte, D., Iturriza, I.J., Biresselioglu, M. E., Demir, M.H., Dimitrova, E., Tasheva, M., Mu- 
tafchiyska, I., Burov, A., Tiberio, L., Panno, A., Carrus, G. \& Costa, S. (2019a) Comparative assessment of European energy lifestyles. Deliverable 5.1, H2020 project ECHOES. Online unter: https:// www.echoes-project.eu/sites/echoes.drupal.pulsartecnalia.com/files/ D5.1\%20\%28R2\%29.pdf (letzter Zugriff: 13.05.2020).

Schwarzinger, S., Kaltenegger, I. \& Bird, D.N. (2019b) „Smarte“ Technologien als Schlüssel zu klimafreundlichem Konsum? In: Hübner, R. \& Schmon, B. (Hrsg.) Das transformative Potenzial von Konsum zwischen Nachhaltigkeit und Digitalisierung. Kritische Verbraucherforschung. Springer VS, Wiesbaden, Deutschland. DOI: https://doi. org/10.1007/978-3-658-26040-8 4

Statistik Austria (2017) Tourism. Bundesanstalt Statistik Österreich, Wien, Österreich. Online unter: https://www.statistik.at/web en/ statistics/Economy/tourism/index.html (letzter Zugriff: 20.06. 2018).

Statistik Austria (2019) STATcube - Statistical Database of STATISTICS AUSTRIA. Datenbanken: Statistiken: Tourismus: Nächtigungsstatistik ab 1974 nach Kalenderjahr. Bundesanstalt Statistik Österreich, Wien, Österreich. Online unter: http://statcube.at/statcube/home (letzter Zugriff: 30.09.2019).

Thaler, R. \& Sunstein, C. (2008) Nudge: improving decisions about health, wealth and happiness. Yale University Press, New Haven, CT, USA.
Umweltbundesamt (2018) Anpassungsaktivitäten der Bundesländer. Umweltbundesamt GmbH, Wien, Österreich. Online unter: http:// www.klimawandelanpassung.at/ms/klimawandelanpassung/de/kwa politik/kwa_bundeslaender/ (letzter Zugriff: 07.09.2018).

UNFCCC (o.J.) Climate: get the big picture. United Nations Framework Convention on Climate Change (UNFCCC). Online unter: https:// bigpicture.unfccc.int/ (letzter Zugriff: 06.09.2018).

Unger, R., Abegg, B., Mailer, M. \& Stampfl, P. (2016) Energy consumption and greenhouse gas emissions resulting from tourism travel in an Alpine setting. Mountain Research and Development 36(4), 475483. DOI: https://doi.org/10.1659/MRD-JOURNAL-D-16-00058.1

Wien Tourismus (2014) Tourismusstrategie 2020. WienTourismus, Wien, Österreich. Online unter: http://tourismusstrategie2020.wien. info/downloads/WT-Tourismusstrategie-2020.pdf (letzter Zugriff: 13.05.2020).

Windsperger, A., Windsperger, B., Bird, D.N., Jungmeier, G., Schwaiger, H., Frischknecht, R., Nathani, C., Guhsl, R. \& Buchegger, A. (2017) Life cycle based modelling of greenhouse gas emissions of Austrian consumption. Publizierbarer Endbericht zum Projekt climAconsum, gefördert durch den Klima- und Energiefonds im Rahmen des Programms „Austrian Climate Research Programme“ (ACRP). Online unter: http://www.indoek.at/downloads/News_2018_climAconsum_ Endbericht.pdf (letzter Zugriff: 12.01.2019).

Open Access Dieses Buch wird unter der Creative Commons Namensnennung 4.0 International Lizenz (http://creativecommons.org/licenses/by/4.0/deed.de) veröffentlicht, welche die Nutzung, Vervielfältigung, Bearbeitung, Verbreitung und Wiedergabe in jeglichem Medium und Format erlaubt, sofern Sie den/die ursprünglichen Autor(en) und die Quelle ordnungsgemäß nennen, einen Link zur Creative Commons Lizenz beifügen und angeben, ob Änderungen vorgenommen wurden.

Die in diesem Buch enthaltenen Bilder und sonstiges Drittmaterial unterliegen ebenfalls der genannten Creative Commons Lizenz, sofern sich aus der Abbildungslegende nichts anderes ergibt. Sofern das betreffende Material nicht unter der genannten Creative Commons Lizenz steht und die betreffende Handlung nicht nach gesetzlichen Vorschriften erlaubt ist, ist für die oben aufgeführten Weiterverwendungen des Materials die Einwilligung des jeweiligen Rechteinhabers einzuholen.

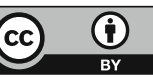

